PHYSICAL REVIEW B 95, 159902(E) (2017)

\title{
Publisher's Note: Coulomb impurities in two-dimensional topological insulators [Phys. Rev. B 95, 125431 (2017)]
}

Jia-Lin Zhu, Guo Li, and Ning Yang

(Received 29 March 2017; published 5 April 2017)

DOI: 10.1103/PhysRevB.95.159902

This paper was published online on 22 March 2017 with an incorrect version of Fig. 2. Figure 2 has been replaced as of 30 March 2017. The figure is correct in the printed version of the journal. 